\title{
New Idea on Exact Solution of the Non Relativistic Schroedinger Equation for a Sextic Potential
}

\author{
Dr. V.K. Srivastava \& (Late) Prof. S.K. Bose \\ Department of Physics D.D.U. Gorakhpur University, Gorakhpur - 273009, U.P., India
}

\begin{abstract}
A useful generalization of an ansatz used to solve non relativistic Schroedinger equation for sextic potential of physical interest such that we obtain a class containing an infinite number of exact solutions for the given potential of physical importance. The exact solution may be used to get the accuracy and the reliability of numerical methods of solving Schroedinger equation.
\end{abstract}

Keywords: Ansatz, Schroedinger equation, sextic potential and exact solution.

\section{Introduction}

One of the important issues of quantum mechanics is to solve the Schroedinger equation for physical potentials. Unfortunately, however, only for few physical potentials e.g. Colomb, harmonic oscillator etc., Schroedinger equation could be exactly solved. Considerable effort has been made is recent years towards obtaining the exact solution of the Schroedinger equation for potential of physical interest. It has recently been shown that the Schroedinger equation for a very large class of physical potentials can be solved by choosing a proper ansatz for the eigenfunction [1-3, 5, 7-10].

In the present work, we wish to point out that a simple generalization of the ansatz used in the paper to obtain the energy eigenvalue and the eigenfunction can give a class of exact solutions of the problem. The class contain an infinite number of solutions. In research paper [6], an exact solution of a three dimensional doubly anharmonic (Sextic) oscillator with central symmetry was obtained whereas in research paper [11], a comment on it was given.

\section{Schroedinger Equation And Exact Solutions}

To find exact solutions of non relativistic Schroedinger equation for various potential of physical interest, consider the Schroedinger equation and is given by

$$
\nabla^{2} \Psi+\frac{2 \mathrm{~m}}{\hbar^{2}}[E-V(r)] \Psi=0
$$

where $\nabla^{2}=$ Laplacian operator

$$
=\left[\frac{1}{r^{2}} \frac{\partial}{\partial^{r}}\left(r^{2} \frac{\partial}{\partial r}\right)+\frac{1}{r^{2} \operatorname{Sin} \theta} \frac{\partial}{\partial \theta}\left(\operatorname{Sin} \theta \frac{\partial}{\partial \theta}\right)+\frac{1}{r^{2} \operatorname{Sin}^{2} \theta} \frac{\partial^{2}}{\partial \Phi^{2}}\right]
$$

$\Psi=$ Normalised wave function

$m=$ mass of particle

$E=$ total energy

$V(r)=$ Potential energy

$\hbar=\frac{h}{2 \pi}$

and $h=$ Planck's constant

The reduced radial part of the Schroedinger equation (1) is given by

$$
\begin{gathered}
-\frac{\hbar^{2}}{2 \mu} \frac{1}{r^{2}} \frac{d}{d r}\left(r^{2} \frac{d R}{d r}\right)+\left\{V(r)-E+\frac{l(l+1) \hbar^{2}}{2 \mu r^{2}}\right\} R(r)=0 \\
V(r)=\left(a^{\prime} r^{2}-b^{\prime} r^{4}+c^{\prime} r^{6}\right) \\
\text { where } a^{\prime}, b^{\prime} \& c^{\prime}=\text { constants } \\
R(r)=\frac{1}{r} \Phi(r) \\
\text { Here } \Phi(r)=\text { New function of } r
\end{gathered}
$$

Suppose

$$
\text { Using equations (4) and (5) in equation(3), we get }
$$

$$
\begin{array}{r}
\frac{d^{2} \Phi}{d r^{2}}+\left\{\lambda-a r^{2}+b r^{4}-c r^{6}-\frac{l(l+1)}{r^{2}}\right\} \Phi(r)=0 \quad \ldots \\
\text { Here } \frac{2 \mu E}{\hbar^{2}}=\lambda, \frac{2 \mu a^{\prime}}{\hbar^{2}}=a, \frac{2 \mu b^{\prime}}{\hbar^{2}}=b \text { and } \frac{2 \mu c^{\prime}}{\hbar^{2}}=c
\end{array}
$$


Let us now generalize the ansatz used earlier i.e.

$$
\Phi(r)=\exp \left[\frac{1}{2} \beta r^{2}-\frac{1}{4} \alpha r^{4}+\delta \log r\right]
$$

to

$$
\Phi(r)=\left[\exp \frac{1}{2} \beta r^{2}-\frac{1}{4} \alpha r^{4}+\log \sum_{n=0}^{\infty} a_{n} r^{2 n+\delta}\right]
$$

Here $\beta, \alpha$ and $\delta$ are constants and are to be determined.

Using equation (9) in equation (6) and setting the coefficient of $r^{2 n+\delta}$ equal to zero, then we have three term recurrence relation,

Where

$$
A_{n-1} a_{n-1}+B_{n} a_{n}+C_{n+1} a_{n+1}=0
$$

Suppose $\quad \alpha^{2}=C$ and $2 \alpha \beta=b$

$$
\begin{aligned}
& A_{n}=\beta^{2}-a-(4 n+2 \delta+3) \alpha \\
& \beta_{n}=\lambda+(4 n+2 \delta+1) \beta \\
& \text { and } C_{n}=(2 n+\delta)(2 n+\delta-1)-l(l+1)
\end{aligned}
$$

Using equations (12) and (7), we have

$$
\alpha=\sqrt{\frac{2 \mu c^{\prime}}{\hbar^{2}}} \text { and } \beta=\sqrt{\frac{2 \mu b^{\prime}}{2 c^{\prime} \hbar}}
$$

Now, for $a_{0}$ to be non zero, we should have

$$
C_{o}=0 \Rightarrow \delta(\delta-1)-l(l+1)=0
$$

$\Rightarrow$ Either $\delta=-l$ or $\delta=(l+1)$

$$
-l \text { or } \delta=(l+1)
$$

in which second value of $\delta$ i.e. $\delta=(l+1)$ is physically acceptable.

A class of exact solutions can be constructed by setting $\mathrm{n}=0,1,2, \ldots \ldots$.

Thus, there are two cases:

Case-I: For $\mathrm{n}=0$, we should have

$$
\begin{aligned}
& B_{0}=0 \Rightarrow \lambda=-(2 \delta+1) \beta=-\left(l+\frac{3}{2}\right) \frac{b}{\sqrt{c}} \\
& \text { and } A_{o}=0 \Rightarrow \beta^{2}-(2 \delta+3) \alpha=a
\end{aligned}
$$

In this case, the normalized eignfunction is given by

$$
R_{l}^{o}(r)=N_{l}^{0} r^{l} \exp \left(\frac{b r^{2}-C r^{4}}{4 \sqrt{c}}\right)
$$

The value of normalization constant $N_{l}^{0}$ was obtained earlier [6].

Case II For $\mathrm{n}=1$, we should have $a_{2}=0$ but $a_{1}=0$

Such that $A_{1}=0 \Rightarrow \beta^{2}-(2 \delta+3) \alpha=a$ and $a_{o} \& a$, satisfy the equation, $A_{o} a_{o}+B_{1} a_{1}=0$

$$
\text { and } B_{o} a_{o}+C_{1} a_{1}=0
$$

For a nontrivial solution, we have

$$
\left|\begin{array}{ll}
A_{o} & B_{1} \\
B_{o} & C_{1}
\end{array}\right|=0
$$

Using equation (11) in equation (22) and on simplifying, we get

gives energy eigenvalues.

$$
\lambda_{ \pm}=-(2 l+5) \beta \pm\left\{(4 l+10) \beta^{2}-(4 l+6)[a+(2 l+5)] \alpha\right\}^{\frac{1}{2}}
$$

$$
\begin{gathered}
\text { and } R_{l}^{1}(r)=N_{l}^{1} r^{l}\left(a_{o}+a_{1} r^{2}\right) \exp \left(\frac{b r^{2}-c r^{4}}{4 \sqrt{c}}\right) \\
\text { gives eigenfunction }
\end{gathered}
$$

provided

$$
a_{1}=-\frac{1}{2(2 l+3)}\left\{-2 \beta \pm\left\{(4 l+10) \beta^{2}-(4 l+6)[a+(2 l+5) \alpha]\right\}^{\frac{1}{2}}\right\} a_{o}
$$

Here $N_{l}^{1}=$ Normalization constant

\section{Results And Discussion}

The exact eigenfunction for different Schroedinger equation for zero energy eigenvalue obtained above can be used to test the accuracy of numerical methods of solving Schroedinger equation. The Schroedinger equation for sextic potential possesses an infinite number of exact solutions. 
Proceeding this way i.e. setting $n=2,3$, we can generate a class containing an infinite number of exact solution. Thus, for a general value of $n$; take $n=p$, then the energy eigenvalue is obtained from the condition

$$
\begin{aligned}
& \Delta_{p+1}=\left|\begin{array}{ccccc}
B_{o} & C_{1} & & 0 \\
A_{o} & B_{1} & C_{2} & & 0 \\
& A_{1} & B_{2} & C_{3} & 0 \\
\ldots & \ldots & \ldots & \ldots & \ldots \\
\ldots & \ldots & \ldots & A_{p-1} & B_{p}
\end{array}\right|=0 \quad \ldots \ldots .(26) \\
& \text { Now, since } a_{p} \neq 0 \Rightarrow a_{p+1}=0 \\
& \Rightarrow A_{p}=0 \Rightarrow \beta^{2}-(2 \delta+4 p+3) \alpha=a
\end{aligned}
$$

In this generalized case, the eigenfunction becomes

$$
R_{l}^{p}(r)=N_{l}^{p} r^{l}\left(a_{o}+a_{1} r^{2}+\ldots . .+q_{p} r^{2 p}\right) \cdot \exp \left(\frac{b r^{2}-c r^{4}}{4 \sqrt{c}}\right) \ldots \ldots
$$

In this genera

in which $a_{i}, i=1,2,3, \ldots . p$ can be expressed in terms of $a_{o}$ using the recurrence relation (10).

\section{Conclusion}

The class containing infinite number of exact solution for sextic potential obtained in this paper can be useful for application in the context of structural phase transition [7], polaron formation in solids [8], the concept of false vacua in field theory [9] and fibre optics [10, 11].

A similar class of exact solutions can be generated by generalizing the ansatz in a similar fashion for multiply anharmonic oscillator with potential like

$$
V(r)=a^{\prime} r^{2}-b^{\prime} r^{4}+c^{\prime} r^{6}-d^{\prime} r^{8}+e^{\prime} r^{10}-f^{\prime} r^{12}+g^{\prime} r^{14}
$$

In future, such generalization can be used to obtain energy eigenvalue and eigenfunction for relativistic Schroedinger equation for multiply anharmonic oscillator potential of physical importance.

\section{Acknowledgement}

The author gratefully acknowledges Prof. H.C. Prasad, Department of Physics, DDU Gorakhpur University, and Gorakhpur for encouragement and giving necessary suggestions.

\section{References}

[2]. Tymczak C J, Japaridze G S, Handy C R and Wang XQ, Phys. Rev. Lett., 80 (1998) 3673.

[3]. Dobrovolska I V and Tutik R S, Int. J. Mod. Phys. A, 16 (2001) 2493

[4]. Dong S H, Physica Scripta, 65 (2002) 289

[5]. Ikhdair S K and Sever R, J. Mol. Struct: THEOCHEM, 806 (2007) 155

[6]. Kaushal R S, Phys. Lett. A, 142 (1989) 57

[7]. $\quad$ Khare A and Behera S N, Pramana ( J. Phys. ), 14 (1980) 327

[8]. Amin D, Phys. Rev. Lett., 36 (1976) 323

[9]. Coleman S, Aspects of symmetry, selected Erice Lectures (Cambridge Univ. Press, Cambridge, 1988) p. 234

[10]. Hashimoto M, Int. J. Electron, 46 (1979) 125, opt. commun., 32 (1980) 383

[11]. Bose S K and Varma N, Phys. Lett. A, 147 (1990) 85 